\title{
The Influence of Gender and Personally Knowing a Victim on Medical Students' Attitudes towards Female Victims of Interpersonal Violence
}

\author{
Madhabika B. Nayak \\ Department of Psychiatry, Faculty of Medicine, Kuwait University, Kuwait
}

\section{Key Words}

Victims of violence - Attitudes - Gender . Medical students

\begin{abstract}
Objective: The purpose of this study was to assess medical students' attitudes towards female victims of interpersonal violence and to examine associations between these attitudes and gender, personally knowing a victim, and knowledge of interpersonal violence issues. Methods: One hundred and two students completed a survey adapted from established attitudinal scales that included an assessment of knowledge of interpersonal violence. Multivariate analyses were conducted using attitude scores as the dependent variables, gender and personally knowing a victim as independent variables, and knowledge of interpersonal violence as a covariate. Results: Both gender and personally knowing a victim had significant associations with attitudes towards victims of vio-
\end{abstract}

\begin{tabular}{ll}
\hline KARGER & (1) 1999 S. Karger AG, Basel \\
Fax +41 61 306 1234 34 & \\
$\begin{array}{l}\text { E-Mail karger@karger.ch } \\
\text { www.karger.com }\end{array}$ & $\begin{array}{l}\text { Accessible online at: } \\
\text { www.karger.com/journals/mpp }\end{array}$
\end{tabular}

lence. Women were found to have lower blaming scores for victims of both rape and domestic violence. With regard to rape victim blaming, personally knowing a victim of interpersonal violence reduced scores for both men and women. However, for domestic violence victim blaming, knowing a victim reduced scores for women only. No significant association was found between knowledge of interpersonal violence and attitudes towards victims of violence. Conclusions: Clinical training that focuses on information alone is unlikely to influence medical student attitudes towards victims of interpersonal violence. Female gender and personally knowing a victim positively influence attitudes towards victims of violence, however the impact of these variables may be specific to the type of violence. Training programs on interpersonal violence must take into account attitudinal differences due to student gender and prior experiences with victims of violence.

Copyright $@ 1999$ S. Karger AG, Basel
Madhabika B. Nayak, PhD

Kas, 2nd floo

Tel. +9111656 8171, Fax +91116852286

E-Mail nayakmb@ndf.vsnl.net.in 
There is significant support in the literature for the association between interpersonal violence and poor health in women [1]. Recent studies, however, indicate that physicians continue to overlook victimization within the context of health care [2,3]. While lack of knowledge about interpersonal violence and specific training in related issues may underlie medical personnel's hesitancy to assess victimization in patients, some investigators have noted that negative attitudes towards victims of violence may also compromise the care received by such victims [3, 4]. However, aside from one study [4] assessing student attitudes regarding female rape victims, little has been documented regarding medical students' knowledge and attitudes regarding violence experienced by women. Given that victims of interpersonal violence are most likely to present to primary care physicians as opposed to psychiatrists and other mental health professionals [5], assessing attitudes of medical trainees is of particular importance. Information on knowledge of and attitudes towards interpersonal violence will help guide development of training programs for medical students.

Several background variables, including gender, personal experience with violence, and level of clinical training may affect student attitudes towards violence against women. Outside the medical literature, studies have documented gender differences in attitudes towards victims, with males being more accepting of forced sex and of myths that blame women for rape [6, 7]. Indeed, some evidence indicates that gender may have a stronger influence on attitudes than race $[8$, 9]. Studies on physicians indicate that women feel more strongly than men about the need for education regarding domestic violence [3].

Research on the role of personally knowing a victim is mixed. Some studies suggest that personally knowing a victim positively influences attitudes towards rape victims. However, this influence may be limited to attitudes held by women only and may not increase empathy for the victim. Unlike the substantial research on effects of gender on attitudes towards violence, the effect of personal experiences with victims on medical student attitudes is just beginning to be empirically explored. DeLahunta and Tulsky [10] noted that physicians may demonstrate reluctance to ask patients about a history of violence because of personal histories. In contrast, medical students who reported histories of abuse were found to more strongly favor education and advocacy roles for physicians [11]. Further, while increasing contact with patients (clinical experience) has not been systematically assessed as a factor influencing student attitudes and knowledge, increased patient contact may impact student attitudes towards victims and perpetrators of violence.

A noteworthy limitation of the research on medical personnel's attitudes is the lack of research in non-Western cultures. Unfortunately, though rape of women reportedly accounted for $36 \%$ of reported violent crimes in Kuwait in 1997 [12], there is a marked absence of literature on violence against women in Kuwait. The objectives of this study were twofold: to assess attitudes towards victims of violence in a sample of medical students in Kuwait and to examine gender, personally knowing a victim, clinical experience and knowledge of interpersonal violence as factors influencing these attitudes.

\section{Methods}

Surveys were distributed among undergraduate medical students from the 4th (preclinical), 5th, and 6th (clinical) years in the summer of 1997. The basic medical degree at the Faculty of Medicine, a Bachelor of Medicine and Bachelor of Surgery (MBBS), takes 
Table 1. Demographic characteristics of study participants by year in medical school

\begin{tabular}{lccc}
\hline & $\begin{array}{l}\text { Preclinical } \\
\text { year 4 } \\
(\mathrm{n}=36)\end{array}$ & $\begin{array}{l}\text { Clinical 1 } \\
\text { year 5 } \\
(\mathrm{n}=36)\end{array}$ & $\begin{array}{l}\text { Clinical 2 } \\
\text { year 6 } \\
(\mathrm{n}=30)\end{array}$ \\
\hline Response rate, \% & 45.9 & 73.5 & 71.4 \\
\hline Age & & & \\
$\quad$ Mean & 21.53 & 23.31 & 24.50 \\
SD & 1.42 & 1.33 & 1.04 \\
Gender, \% male & 55.6 & 38.9 & 32.3 \\
Marital status, \% single & $\begin{array}{c}\text { (unmarried or divorced) } \\
\text { Male }\end{array}$ & 78.6 & 55.6 \\
Female & 81.3 & 72.3 & 71.4 \\
\end{tabular}

Number of students enrolled in the year 1996-1997 in years 4, 5, and 6 were 87,49 , and 42 , respectively; computation of response rates includes incomplete surveys that were excluded from final analyses that used data from 102 respondents.
7 years of training; clinical training commences in the 5 th year and residency is completed in the 7 th year. A total of 106 students returned the surveys, an overall response rate of $60 \%$. The response rate for clinical year (5th and 6th year) students was significantly higher than that for the preclinical year students $\left(\chi^{2}=\right.$ $17.68, \mathrm{p}<0.001$; table 1). The final sample comprised of 102 students divided somewhat evenly across 3 years and was similar in age and gender distribution (42.2\% male; $57.8 \%$ female) to that of the student body at large (students enrolled in years 4 through 6 for the year 1996-1997 were $42.1 \%$ men, $57.9 \%$ women).

The survey, approved by the research committee of the Faculty of Medicine, was a paper-and-pencil questionnaire that comprised of three sections. The first section provided information on background (demographic) variables including year of training, clinical rotations completed, and items assessing personal experience with victimization. Due to discomfort of the research review committee (secondary to perceptions of violence being considered taboo in the Arab culture), students' personal experiences with interpersonal violence were not assessed directly. Instead they were asked if they had personally known a victim of violence (rape, domestic violence, and child abuse) and if they had ever witnessed adult family members hit or physically attack each other. Students were also asked if they had seen patients who had reported being victimized by interpersonal violence.
The second section of the survey assessed attitudes towards victims and perpetrators of rape and domestic violence using 19 items with a 4-point Likert-type scale with options ranging from strongly agree to strongly disagree. Items indicated were adapted from previously established scales on rape and domestic violence attitudes [13, 14]. Responses to items of the survey were averaged to provide separate victim blame scores for rape and domestic violence. These scores ranged from 1 to 4 with increasing scores indicating a greater tendency to blame the victim for the violence.

The last section of the survey comprised of 14 items assessing knowledge of characteristics, prevalence, and sequelae of rape and domestic violence. These items could be answered true/false; the 7 items on domestic violence were obtained from a published survey, and the remaining 7 items on sexual assault were designed by the author. An overall knowledge score was computed by adding the number of correct answers and could range from 0 to 14 .

A Multivariate Analysis of Covariance (MANCOVA) was conducted to examine associations among the attitude scores, gender, personally knowing a victim, and knowledge of interpersonal violence. This analysis was conducted using the attitude scores as dependent variables, gender and personally knowing a victim as independent variables and the total knowledge score as the covariate. 
Fig. 1. Rape victim blame score by gender and personally knowing a victim.

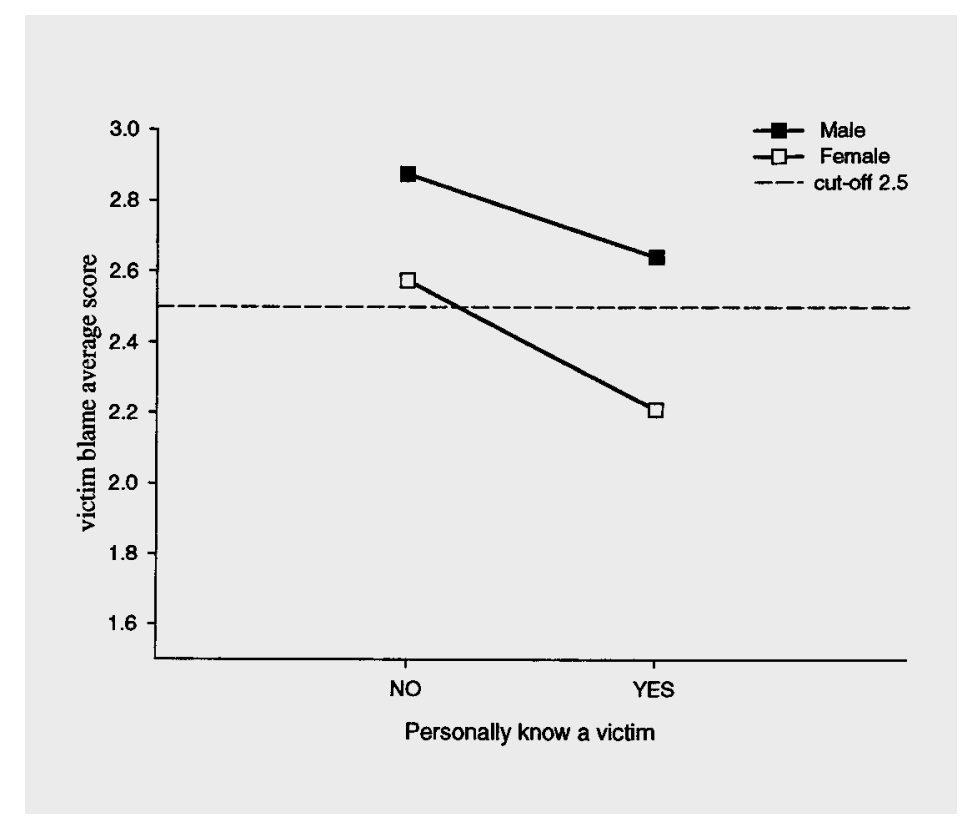

\section{Results}

Exposure to Interpersonal Violence Issues via Personal Knowledge of a Victim, Witnessing Adult Violence, and Contact with Patients

Overall $34.3 \%$ of the students reported personally knowing an individual who had been raped, experiences domestic violence, or child physical or sexual abuse. Over half of all students $(54.9 \%)$ reported having witnessed adult family members physically attack each other. Clinical experience with a victim of interpersonal violence was infrequently reported. Percentages of students who reported having seen patients reporting rape, domestic violence, or both rape and domestic violence were $6.9,4.9$, and $3.9 \%$, respectively; $2.9 \%$ reported seeing child patients who had experienced abuse.

Medical Student Attitudes
Attitudes towards Victims and Perpetrators and Knowledge of Interpersonal Violence

Victim blame scores ranged from 1 to 4 with a score of 2.5 or greater indicating a tendency to blame the victim for the violence. Overall, $64.7 \%$ of the sample scored at 2.5 or higher on victim blaming for rape, $56.9 \%$ obtained a score of 2.5 on victim blaming for domestic violence. There were no significant differences in attitude scores by year of medical training, contact with patients who reported victimization, or witnessing adults physically attack each other. Hence these variables were not included in the final analyses on the attitude scores. With regard to knowledge of interpersonal violence, $54.9 \%$ of the students obtained total knowledge scores of 7 or less (out of 14). The total knowledge score was significantly correlated with the domestic violence victim blame scores $(-0.30, \mathrm{p}<0.01)$ but not with the rape victim blame score. 


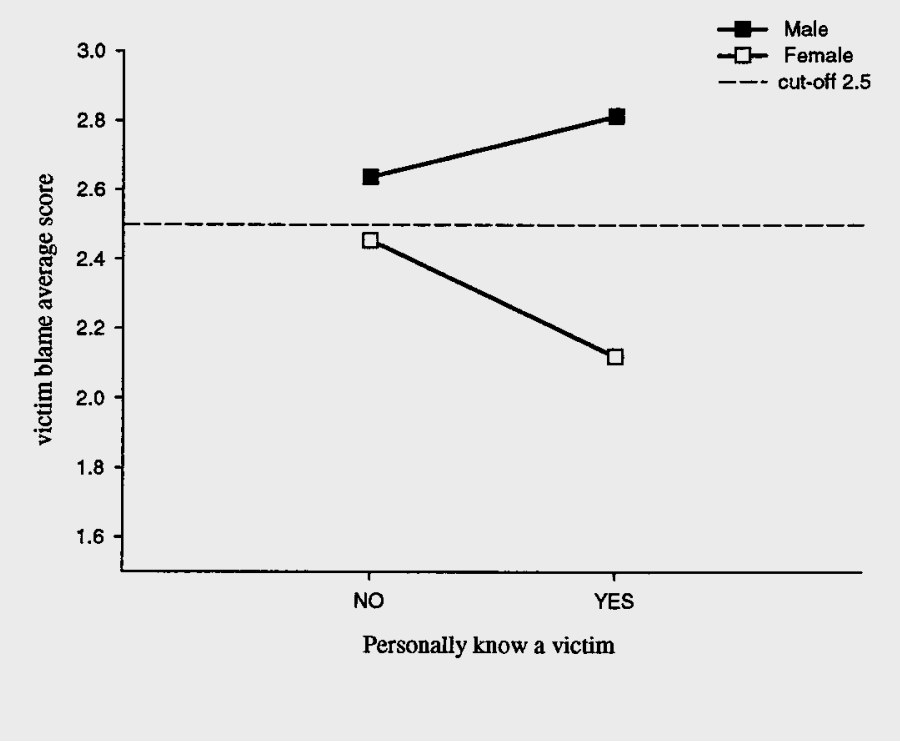

2. Domestic violence victim blame score by gender and personally knowing a victim.

Factors Influencing Attitudes towards Victims and Perpetrators of Interpersonal Violence

The overall model was significant for both the attitude scores, however knowledge was not significant as a covariate. Both gender and personally knowing a victim had significant independent effects on the rape victim blame score. Women had a lower average rape victim blame score $($ mean $=2.44, \mathrm{SD}=0.45)$ compared to men (mean $=2.80, \mathrm{SD}=0.45$, $\mathrm{p}<0.01$ ). Both men and women students who personally knew a victim obtained lower rape victim blame scores (mean $=2.36, \mathrm{SD}=0.49$ ) compared to those who did not personally know a victim $($ mean $=2.71, \mathrm{SD}=0.44, \mathrm{p}<$ $0.01)$. For the domestic violence victim blame score, a significant interaction was found for gender and personally knowing a victim. Specifically, personally knowing a victim significantly reduced domestic violence blame scores for women alone. Women who knew a victim had a lower average domestic violence blame score (mean $=2.12, \mathrm{SD}=0.50)$ than women who had not known a victim personally $($ mean $=2.45, \mathrm{SD}=0.39, \mathrm{p}<0.01)$. Figures 1 and 2 present mean attitude scores as a function of gender and personally knowing a victim.

\section{Discussion}

This is the first study to examine the influence of gender, personally knowing a victim, and knowledge of interpersonal violence on the attitudes of medical students in Kuwait towards female victims of violence. The results suggest that while gender and personally knowing a victim influence attitudes towards victims of violence, increase in medical training and theoretical knowledge of issues related to interpersonal violence do not have a significant impact on attitudes towards victims. 
A similar lack of impact of formal education and exposure to knowledge and experience in child maltreatment on personal attitudes regarding child abuse has been reported for social science students in Palestine [15]. Taken together, these findings suggest that standard clinical training does not change attitudes towards victims of violence. Needless to say, training in issues of interpersonal violence is indicated for medical students.

Information on factors that influence attitudes towards victims of violence is of great importance in guiding development of training programs. Consistent with previous research, the present findings suggest that, compared to male medical students, female students are less likely to blame victims of rape and domestic violence. Personally knowing a victim of violence appears to differentially impact attitudes, based on gender and type of violence. Previously, Anderson et al. [9] had noted that the influence of personally knowing a rape victim on attitudes may be limited to women. Similarly in our study, personally knowing a victim reduced blaming of domestic violence victims for women only. Personally knowing any victim of violence appears to reduce blame on a rape victim for both women and men in the study. However, it should be noted that the average rape victim blame score for men who reported knowing a victim remained above the cutoff of 2.5 (indicating a tendency to place blame on the victim), while that for women reporting personally knowing a victim fell to below the cutoff of 2.5 (fig. 1). The importance of assessing different dimensions of attitudes towards victims of violence (e.g. type of violence, degree of blaming) is exemplified by these findings.

While there are few published studies on the prevalence of violence against women in middle-eastern countries, some reports suggest it is a significant health issue. Data from Alexandria, Egypt, indicated that domestic violence accounts for $28 \%$ of all visits by women to medical trauma units [1]. One in 5 women attending outpatient obstetric-gynecological services in Kuwait reported a lifetime history of criminal assaults when asked specifically about various stressful events [16]. Sequelae of abuse reported are similar in cultures as diverse as the US, Kenya, Chile, and Kuwait [17]. Hence, violence against women is a health issue that cannot be overlooked by health professionals anywhere in the world.

The overall moderate response rate (particularly the high nonresponse rate of preclinical students) to this study may reflect medical students' inhibitions in addressing issues of interpersonal violence and underlying negative attitudes towards victims of violence. However, despite the limitation of a relatively high nonrespondent rate, this study provides important data on medical student attitudes towards victims of violence on which further studies may be designed. If physicians hold negative attitudes towards victims, they may be less likely to assess victimization as part of medical care and less likely to offer help for medical ailments that may be consequences of victimization. Medical students are unlikely to benefit from training that simply provides information on the nature and consequences of interpersonal violence. Previous research on nonmedical service providers, such as police [18], has shown that trainees with the least negative and stereotypical perceptions of victims are most likely to benefit from training and implement the training in their work.

\section{Conclusion}

This study illustrates the need to systematically assess different dimensions of medical students' attitudes towards female victims of interpersonal violence. The lack of associa- 
tion of theoretical knowledge of interpersonal violence and of increasing medical training on student attitudes supports the need for training in issues of interpersonal violence as an integral part of medical education. It is crucial that we systematically address attitudes of health care providers as a part of this training.

Training in interpersonal violence issues must address gender differences in attitudes. In order to increase empathy for victims, it may also be important to process (possibly painful and confusing) prior experiences that trainees may have had with victims of violence. If we are to help medical trainees work better with victims of violence, myths that blame the victim for the violence and its health consequences need to be dispelled. Only then can barriers to the provision of optimal health care for victims of violence be removed.

\section{References}

1 Heise L, Raikes A, Watts C, Zwi A: Violence against women: A neglected public health issue in less developed countries. Soc Sci Med 1994;39:1165-1179.

2 Parsons LH, Zaccaro D, Wells B, Stovall TG: Methods of and attitudes towards screening obstetrics and gynecology patients for domestic violence. Am J Obstet Gynecol 1995;173:381-387.

3 Easteal PW, Easteal S: Attitudes and practices of doctors toward spouse assault victims: An Australian study. Violence Vict 1992;7:217228.

4 Best CL, Dansky BS, Kilpatrick DG: Medical students' attitudes about female rape victims. J Interpersonal Violence 1992; 7:175-188.

5 Kimerling R, Calhoun K: Somatic symptoms, social support, and treatment seeking among sexual assault victims. J Consult Clin Psychol 1994;62:333-340.

6 Davis TC, Peck GQ, Storment JM: Acquaintance rape and the high school student. J Adolesc Health 1993;14:220-224.
7 Falchikov N: Adolescent attitudes to abuse of women: Are wives and non-marital partners viewed differently? J Interpersonal Violence 1996;11:391-409.

8 Kalof L, Wade BH: Sexual attitudes and experiences with sexual coercion: Exploring the influence of race and gender. J Black Psychol 1995; 21:224-238.

9 Anderson L, Stoelb MP, Duggan P, Hieger B, Kling KH, Payne JP: The effectiveness of two types of rape prevention programs in changing the rape-supportive attitudes of college students. J College Student Dev 1998;39:131-142.

10 DeLahunta EA, Tulsky AA: Personal exposure of faculty and medical students to family violence. JAMA 1996;275:1903-1906.

11 Cullinane PM, Alpert EJ, Freund KM: First-year medical students' knowledge of, attitudes toward, and personal histories of family violence. Acad Med 1997;72:48-50.

12 Arab Times, Kuwait: Who's bad? Aug 2, 1998, pp 1, 4 .
13 Burt MR: Cultural myths and supports for rape. J Pers Soc Psychol 1980;38:217-230.

14 Harway M, Hansen M: Spouse Abuse: Assessing and Treating Battered Women, Batterers, and Their Children. Sarasota, Professional Resource Press, 1993.

15 Haj-Yahia MM: Child maltreatment as perceived by students of social science in the West Bank. Child Abuse Negl 1995;19:12091219.

16 Nayak MB, Al-Yattama M: Assault history as a factor in depression during pregnancy. Obstet Gynecol 1999;94:204-208.

17 United Nations: Violence against Women in the Family. New York, United Nations Publication, 1989.

18 Campbell R: The role of work experience and individual beliefs in police officers' perceptions of date rape: An integration of qualitative and quantitative methods. Am J Community Psychol 1992;23:249_ 277. 